\title{
New Surgical Approach To Overcome The Inability Of Injured Mammalian Axons To Grow Within Their Environment
}

\author{
Arieh Solomon, Vered Lavie*, Sjana Ben-Bassat, Michael Belkin and Michal Schwartz* \\ Goldschleger Eye Research Institute, Sackler School of Medicine, Tel Aviv University, \\ Tel Hashomer, Israel and *Department of Neurobiology, \\ The Weizmann Institute of Science, Rehovot 76100, Israel
}

\begin{abstract}
SUMMARY
We present a new method for creating conditions conducive to axonal growth in injured optic nerves of adult rabbits. The surgical approach consists of making a cavity in the adult rabbit optic nerve, into which a piece of nitrocellulose soaked with conditioned medium originating from regenerating fish optic nerves is implanted. In addition, daily irradiation (10 days, $5 \mathrm{~min}, 35 \mathrm{~mW}$ ) with low energy $\mathrm{He}-\mathrm{Ne}$ laser is carried out. Such a combined treatment may open a door to neurobiologists and clinicians, hoping to unravel the enigma of mammalian CNS regeneration.
\end{abstract}

Key words: Regeneration; mammalian CNS; surgical approach

\section{INTRODUCTION}

Mammalian central nervous system (CNS) neurons have a negligible capacity to regenerate after lesions $15,6,8,12 /$. In contrast, central nervous system neurons of lower vertebrates reliably regenerate after axotomy. In the goldfish visual system, the retinal ganglion cells regenerate severed axons which become myelinated and form their normal pattern of synaptic functional

Reprint address:

Prof. Michal Schwartz

Department of Neurobiology

The Weizmann Institute of Science

Rehovot 76100, Israel contacts with their target /11/. Optic nerve injury in mammals results in a failure of the surviving cells to regrow their axons and consequently to death of most of the axotomized neurons. Studies carried out by several research groups $/ 1,16 /$, as well as by the group of Schwartz et al. /7,13/, have led to the prevailing hypothesis that axotomized neurons, mammalian as well as nonmammalian, are capable of regenerating their injured axons, but the success or failure of the regeneration process depends upon the response of the nonneuronal cells (astrocytes, oligodendrocytes, microglia cells) to the injury $/ 1,7,13 /$. Accordingly, it is reasonable to assume that immediately after injury, neurons enter a mode of growth which will be expressed only if the environment of these axons is simultaneously conducive for axonal growth.

In a system which spontaneously regenerates after injury, like fish optic nerve, the nonneuronal cells which surround the injured axons create an environment which is conducive for growth. We have shown that such a permissive environment is, at least partially, the result of the presence of soluble substances which, when applied to a nonregenerative system such as adult rabbit optic nerve, have a beneficial effect on growth $/ 3,4,7,9,10,13,17 /$. The challenge which the surgeon has to meet in promoting growth of the injured rabbit optic axons is in finding the most effective yet minimally injurious technique by which to present the injured rabbit axons with the soluble substances promoting regeneration.

The surgical approach that we currently use to obtain growth in injured adult nonregenerative CNS has been developed over the past few years. Our previously reported results showed that the surgical method of applying soluble sub- 
stances, originating from regenerating fish optic nerve, used by us at that time resulted in limited growth $/ 9 /$. It has also been previously shown by us that irradiation with low energy He-Ne laser slows down post-traumatic degeneration /2,14/. Moreover, we have found that laser irradiation combined with the application of soluble substances leads to the most significant growth of normally nonregenerative CNS. Initially, we tried to apply the soluble substances by implanting a segment of regenerating fish optic nerve between the stumps of transected adult rabbit optic nerves, but immunological rejection occurred /15/. We also tried a "wrap around" silicone tube containing soluble substances, originating from the fish optic nerves, in the form of conditioned medium (CM) $/ 15 /$. The silicone tube placed some mechanical pressure on the nerve, so we have since substituted it with a method, discussed here, consisting of insertion of a piece of nitrocellulose soaked in CM into the nerve, that is a preferable means of application.

\section{MATERIALS AND METHODS}

\section{Transorbital exposure of rabbits' optic nerve}

All surgery was carried out while the rabbits were deeply anesthetized by $5 \mathrm{mg} / \mathrm{kg}$ xylazine and $35 \mathrm{mg} / \mathrm{kg}$ ketamine $\mathrm{HCl}$ administered subcutaneously.

Minimal trauma while exposing the optic nerve is essential for successful subsequent growth induction, that can be achieved by the following method, which is modified and based on our previously described approach /15/. Lateral cantotomy, $10 \mathrm{~mm}$ in length, is performed. The conjunctiva is opened along the temporal limbus from 12 to 3 o'clock. Careful attention is given to the very large venous sinuses lying within the orbital cavity at its rim surrounding the eyeball like a ring. A small gap in that ring exists at the 2 o'clock position through which we approach the orbital cavity. This approach is crucial in preventing damage to the sinuses which may otherwise bleed and severely disturb the operation. The optic nerve is reached in the following manner (Figure 1): The superior rectus muscle (SR) is isolated and cut 3

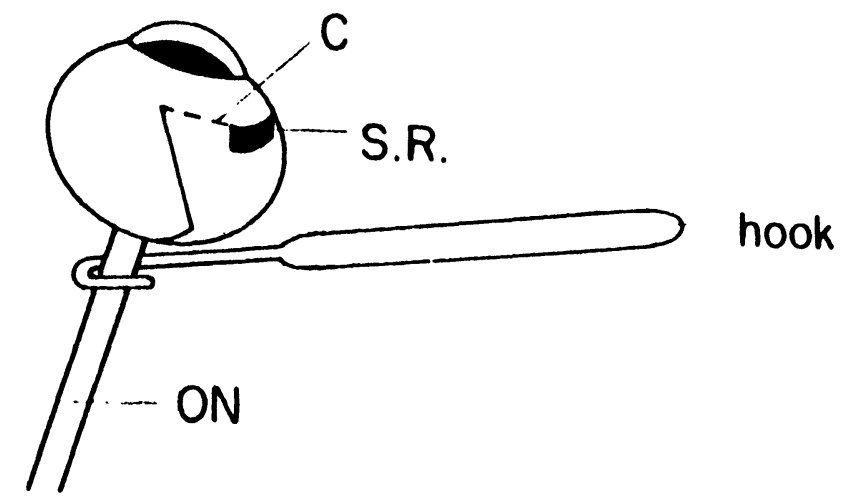

Fig. 1: The eye globe and the optic nerve of rabbit engaged by the muscle hook. C - opening line of conjunctiva; S.R. - superior rectus muscle stump; ON-optic nerve.

$\mathrm{mm}$ from its scleral insertion. The underlying superior oblique muscle is isolated and dissected from its insertion. The superior rectus stump is grasped with fixation forceps and is used to rotate the globe. Then the orbital contents, mostly fat, are cleaned away and pushed to the orbital wall. The retractor bulbi muscles then become visible and are cut. The optic nerve is cleaned and exposed and its point of emergence from the globe is located. A muscle hook is introduced into the space created between the globe and the orbital wall. The edge of the hook is directed to the point of emergence of the optic nerve on its right side. The hook is moved counterclockwise around the nerve until its tip comes out on the left side of the optic nerve. The globe and the optic nerve are now engaged on the hook (Figure 1). The whole length of the optic nerve can be exposed down to the optic foramen and is now ready for treatment. At the end of the treatment, the globe is replaced back in position, and the conjunctiva is closed along the limbus by three 6-0 adsorbable sutures. One of these sutures is used to fix the conjunctiva to the superior rectus muscle stump, in order to avoid extensive downward rotation of the eye. The cantotomy is closed by two 6-0 adsorbable 
sutures. Local antibiotics are applied to the eye for two consecutive days (sulfacetamide 10\% drops and chloramphenicol 5\% ointment). The procedure described above can be performed with almost no bleeding, minimal damage to the orbital structures and no postoperative complications.

\section{Implantation of diffusible substances into the} optic nerve of adult rabbit

Currently, the method used to apply the diffusible substances to the site of injury is as follows: The nerve is exposed as described above. Microsurgical scissors are used to create a longitudinal $1 \mathrm{~mm}$ opening through the meninges, at a distance of $5 \mathrm{~mm}$ from the point of emergence of the optic nerve from the globe. A tuberculine syringe with a $27 \mathrm{G}$ needle is used to create a cavity in the optic nerve tissue. The needle is bent at two places: at its tip and $4 \mathrm{~mm}$ from its base (Figure 2b). The needle's bent tip is introduced into the nerve through the previously created opening in the meninges and is rotated clockwise and counterclockwise excising out neural tissue from the nerve, leaving the meninges intact. At this stage, a triangular
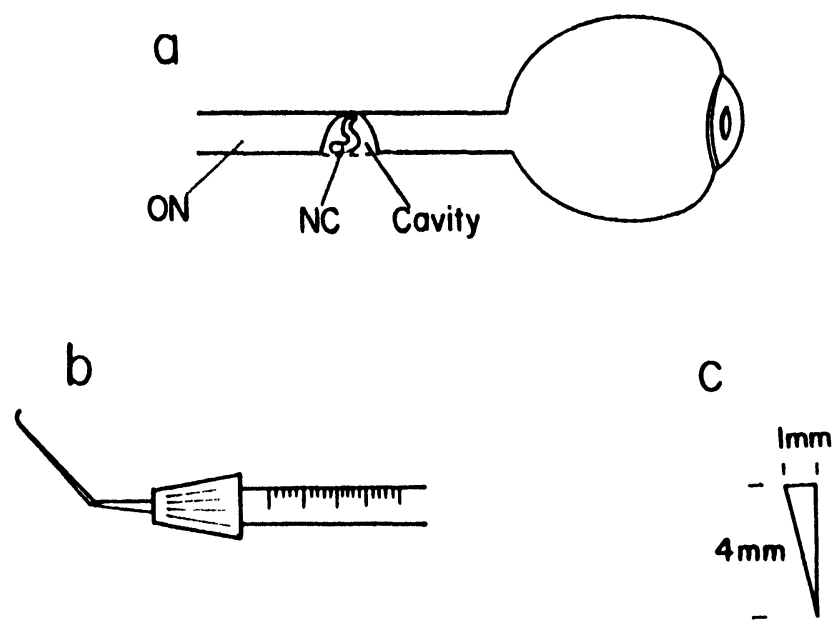

Fig. 2: (a) Cavity created in the rabbit optic nerve into which the nitrocellulose (NC) is introduced and folded upon insertion; (b) a $27 \mathrm{G}$ needle bent at its base and tip used for cavity creation; (c) the size and shape of the nitrocellulose segment. piece of nitrocellulose paper 3-4 $\mathrm{mm}$ in length, which was previously soaked for $15 \mathrm{~min}$ at room temperature in the diffusible substances, is introduced into the created cavity of the nerve (Figure 2c). The nitrocellulose paper folds within the cavity created in the optic nerve (Figure 2a). The nitrocellulose is being used as a vehicle for any substance implanted into the optic nerve. After the implantation, the eyeball is replaced in position and the conjunctiva and canthus closed, as previously described.

According to our treatment regimen, the nerves are irradiated transorbitally $5 \mathrm{~min}$ daily with a $35 \mathrm{~mW}$ He-Ne laser (632.8 nm Spectra Physics Stabilite, model 124B) for 10 consecutive days. The laser beam, with a diameter of 1.1 $\mathrm{mm}$, is focused by a mirror (Physik Instrumente) on the central axis of the cornea. The irradiation penetrates through the eye and the optic disc, up to a distance of $1 \mathrm{~cm}$ behind the globe. He-Ne laser irradiation is applied at a dosage that was found optimal in terms of power, duration of treatment and number of consecutive treatments $/ 2 /$. Assessment of the results was done mainly by electron microscopy, since this is the only method that permits a reliable distinction between surviving axons that escaped the lesion and newly growing axons, supposed to be regenerating. Moreover, electron microscopical analysis allows the tracing of axons having a narrower diameter than can be observed by light microscopy. The results observed by electron microscopical analysis, as well as the immunohistochemical results were described elsewhere /10/.

\section{RESULTS AND DISCUSSION}

The method of surgery discussed above serves as a model for testing the effect of potential therapeutic soluble substances on the neural reaction to injury. It is a relatively simple, short operation and has certain advantages: (i) there is very little bleeding during the surgery, as the venous sinuses lining the anterior orbital rim in the rabbit are avoided; (ii) the optic nerve meninges are minimally disturbed and, thus, the physical continuity of the nerve itself is maintained, requiring no sutures which might 

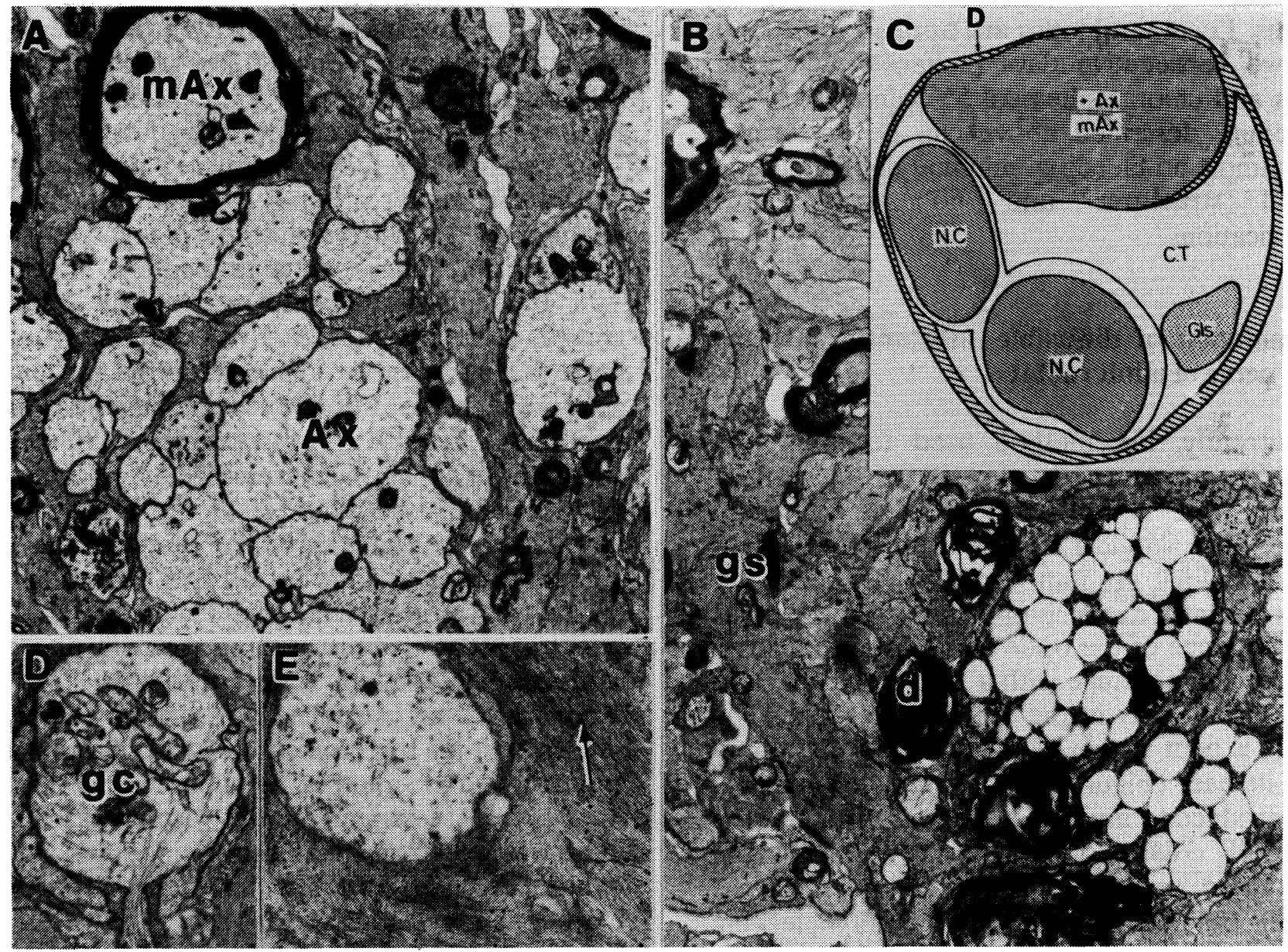

Fig. 3: Analysis by EM of injured treated and untreated nerves. Rabbit optic nerves were transected at a distance of $5 \mathrm{~mm}$ from the eyeball. In the treated nerve, a piece of nitrocellulose $(1 \mathrm{~mm} \times 4 \mathrm{~mm})$ soaked with medium conditioned by regenerating fish optic nerves $(100 \mu \mathrm{g}$ protein $/ \mathrm{ml})$ was placed in the cavity formed by the transection. Beginning $30 \mathrm{~min}$ after surgery, the rabbits were irradiated transocularly for 10 days with low energy, He-Ne laser irradiation ( $630 \mathrm{~nm}, 5 \mathrm{~min}, 35 \mathrm{~mW}$ ). In the control injured nerves, the CM was not applied and the laser treatment was omitted. Eight weeks after surgery, the nerves were dissected out and fixed with $1 \%$ paraformaldehyde and $2.5 \%$ glutaraldehyde in $0.1 \mathrm{M}$ cacodylate buffer $\mathrm{pH} 7.4$. The excised nerves were divided into $1 \mathrm{~mm}$ segments, processed for electron microscopy, and embedded in polybed (Polyscience). Thin sections from each region were mounted on 200 mesh grids: (C) shows a diagram of the cross-section of an injured treated nerve dissected out 8 weeks after injury, which was used for quantitative analysis that resulted in 14068 counted axons (see Results) in the compartment designated $A x+m A x$. The diagram was constructed from a photomontage prepared from low magnification electron micrographs; $\mathrm{NC}$ - nitrocellulose; $\mathrm{CT}$ - connective tissue; $\mathrm{mAx}+\mathrm{Ax}$ - compartment containing viable myelinated (mAX) and unmyelinated $(A X)$ axons; Gls - compartment containing glial scar (Gls). The photograph in $(A)$ was taken from a compartment designated by $m A x+A x$ of a 6-week postoperated treated nerve, and shows a myelinated axon ( $\mathrm{MAX}$ ) and about 23 unmyelinated axons (AX) embedded in astrocytic processes (Ap); (D) shows a growth cone (gc) embedded in astrocytic processes. Micrograph (E) shows unmyelinated axons embedded in astrocytic processes labeled with antibodies directed to GFAP (rabbit anti-GFAP, Sigma), using postembedding immunocytochemistry with gold labeled second antlbodies (15 $\mathrm{nm}$, Jenseen, Sweden). The arrow shows gold particles indicative of GFAP positive immunoreactive sites. Micrograph of control injured nontreated animal (B) shows an extensive glial scar (gs) in which some degenerating axons (d) are embedded. In these experiments, the surgery and the processing of the tissue were carried out by two different people; animals were coded and the codes were not broken until the final analysis of the experiments. (A,B) $\times 6580$; (D) $\times 11375$; $(E) \times 16835$. 
alter the healing process; (iii) the operation is carried out under direct surgical microscope observation, taking special care to avoid the blood vessels in the vicinity of the optic nerve and, thus, the blood supply to the eyeball can be minimally disturbed; and (iv) the nitrocellulose paper causes no or little inflammatory reaction in the surrounding tissues. This method of introducing materials to the injured nerves, however, has a drawback in that the nitrocellulose occupies part of the nerve's volume. It thus affects the healing process physically, since potential regrowing axons cannot grow through the nitrocellulose.

The surgical method described above was used by us to injure adult rabbit optic nerves and apply to them soluble substances originating from regenerating fish optic nerves in the form of CM. When this method was combined with transocular irradiation by a low energy $\mathrm{He}-\mathrm{Ne}$ laser, it led to considerable regrowth of severed optic nerve axons. The nerves were analyzed using electron microscopy. Such an analysis revealed, for example, in the injured treated nerves, 6 weeks post-injury, many viable axons distal to the site of injury (Figure 1A). In contrast, in the control injured but not treated nerves, no myelinated or unmyelinated viable axons were found distal to the site of the injury (Figure 3B). Many of the viable axons observed in the treated injured nerves distally to the site of injury were nonmyelinated and thinly myelinated (Figure 3A). Therefore, these axons can be considered to be newly growing axons rather than spared by the injury. The conclusion that those are newly growing nerves was supported by the presence of growth cones (Figure 3D; see also the results described by Lavie et al., 1990). These axons originated from the retinal ganglion cells, as shown by injecting horseradish peroxidase intraocularly $/ 10 /$. The observed growing axons were embedded in cellular elements of CNS environment such as astrocytes. This was evident by immunocytochemical staining using antibodies directed against the astrocytic intermediate filament, the glial fibrillary acidic protein (Figure 3E; see also the results in Lavie et al., 1990). A complete description of the evidence that the treatment described above leads to axonal growth in the adult mammalian otherwise nonregenerating nerve is given elsewhere $/ 10 /$. We have also counted the concrete number of axons which traversed the site of injury in injured treated nerves, 8 weeks after injury. These axons were compartmentalized in one compartment, designated $A x+m A x$, shown in the scheme in Figure 3C. The scheme represents the cross-section through the nerve, at a distance of $1.5 \mathrm{~mm}$ distal to the site of the injury. Such counting resulted in 14068 axons.

\section{ACKNOWLEDGEMENTS}

This work has been supported by the Daniel Heumann Fund for Spinal Cord Injury, and by the US Army Medical Research and Acquisition Activity, to M.S.

\section{REFERENCES}

1. Aguayo AJ, David S, Richardson P, Bray G. Axonal elongation in peripheral and central nervous system transplantations. Adv Cell Neurobiol 1978;3:215-221.

2. Assia E, Rosner M, Belkin M, Solomon A, Schwartz M. Temporal parameters of low energy laser irradiation for optimal delay of post-traumatic degeneration of rat optic nerve. Brain Res 1988;476:205-212.

3. Cohen A, Schwartz M. Characterization of primary glial cells from regenerating fish optic nerve. J Neurochem (Suppl) 1989;52:51.

4. Cohen A, Sivron T, Duvdevani R, Schwartz $M$. Regeneration of adult optic nerve is associated with oligodendrocyte maturation inhibitory factor. Neurosci Abstr 1989;15:1259.

5. Grafstein B, Ingoglia NA. Intracranial transection of the optic nerve in adult mice: Preliminary observations. Exp Neurol 1982;76:318-330.

6. Guth L, Windle WF. Regeneration in the vertebrate central nervous system. Exp Neurol 1970;5:1-43.

7. Hadani M, Harel A, Solomon A, Belkin M, Lavie V, Schwartz M. Substances originating from the optic nerve of neonatal rabbit induce regenerationassociated response in the injured optic nerve of adult rabbit. Proc Natl Acad Sci USA 1984;81:7965-7969.

8. Kiernan JA. Hypotheses concerned with axonal regeneration in the mammalian nervous system. Biol Rev 1979;54:155-197.

9. Lavie V, Harel A, Doron A, Solomon A, Lobel D, Belkin M, Ben-Bassat S, Sharma S, Schwartz M. Morphological response of injured adult rabbit optic nerve to implants containing media conditioned by 
growing optic nerves. Brain Res 1987;419:166-173.

10. Lavie V, Murray M, Solomon A, Ben-Bassat S, Rumelt S, Schwartz M. Growth of injured rabbit optic axons within their degenerating optic nerve. J Comp Neurol 1990; 298: 293-314.

11. Murray M. Regeneration of retinal axons into the goldfish optic tectum. J Comp Neurol 1976;168:175196.

12. Ramón y Cajal S. Degeneration and Regeneration of the Nervous System, Vol 1, May RM, transl. New York: Hafner Publ Co, 1959.

13. Schwartz M, Belkin M, Harel A, Solomon A, Lavie V, Hadani M, Rachailovich I, Stein-Izsak C. Regenerating fish optic nerves and a regenerationlike response in injured optic nerves of adult rabbits. Science 1985;228:600-603.

14. Schwartz M, Doron A, Erlich M, Lavie V, Ben-Bassat
S, Belkin M, Rochkind S. Effect of low-energy He-Ne laser irradiation on post-traumatic degeneration of adult rabbit optic nerve. Lasers Surg Med 1987;7:5155.

15. Solomon A, Belkin M, Hadani M, Harel A, Rachailovich I, Lavie V, Schwartz M. A new transorbital surgical approach to the rabbit's optic nerve. J Neurosci Methods 1985;12:259-262.

16. Vidal-Sanz M, Bray MB, Villegas-Pérez MP, Thanos $\mathrm{S}$, Aguayo AJ. Axonal regeneration and synapse formation in superior colliculus by retinal ganglion cells in the adult rat. J Neurosci 1987;7:2894-2909.

17. Zak N, Harel A, Bawnik Y, Ben-Bassat S, Vogel Z, Schwartz M. Laminin immunoreactive sites are induced by growth associated triggering factors in injured rabbit optic nerve. Brain Res 1987;408:263266. 

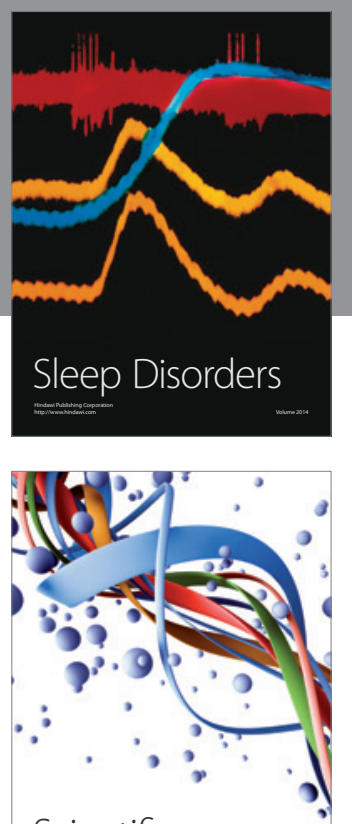

Scientifica
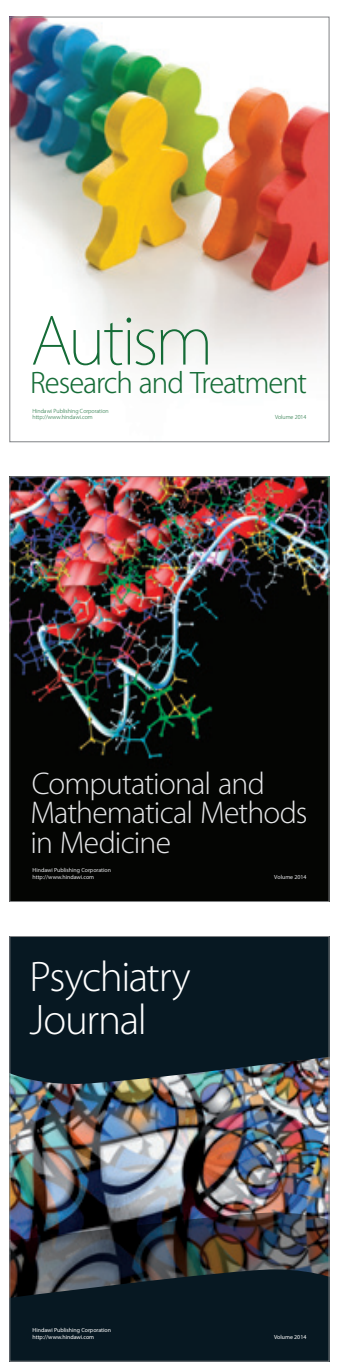
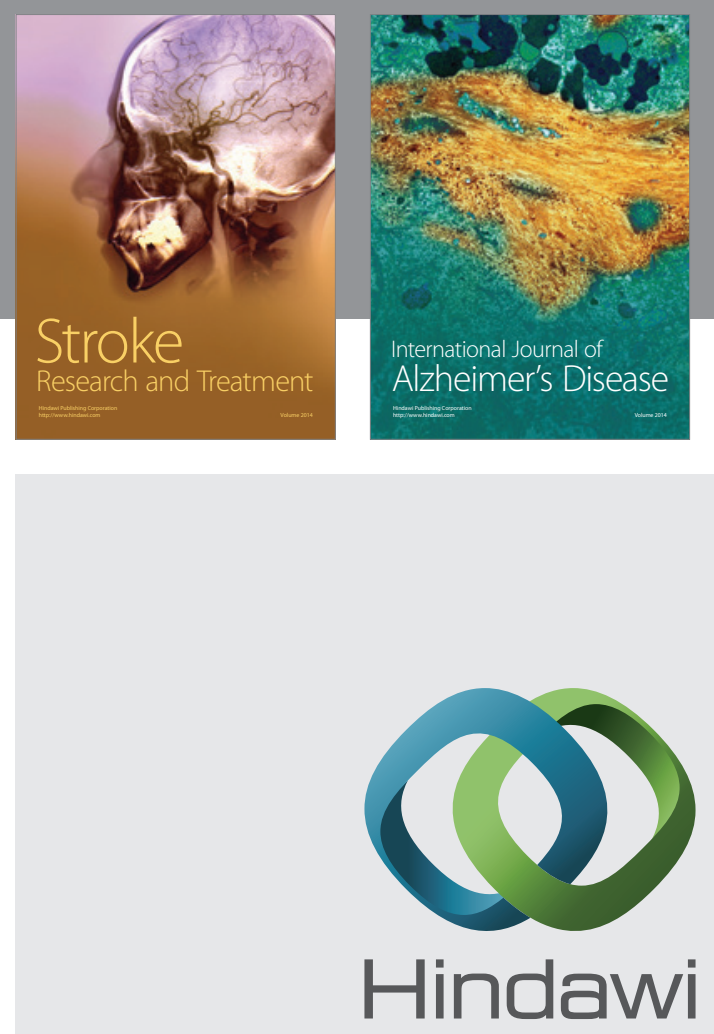

Submit your manuscripts at

http://www.hindawi.com
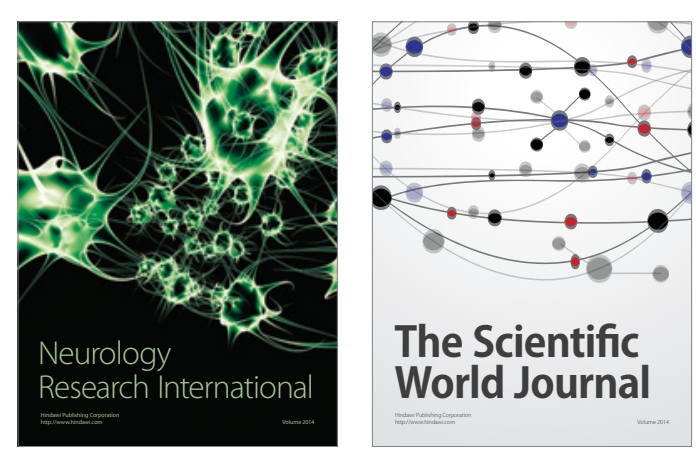

The Scientific World Journal

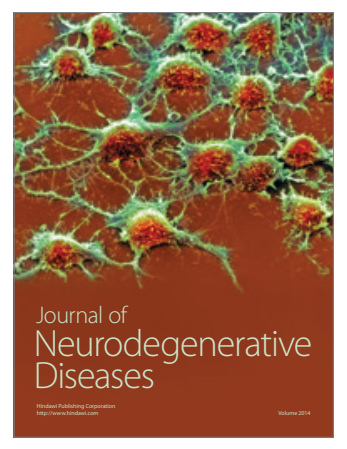

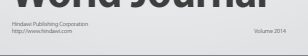

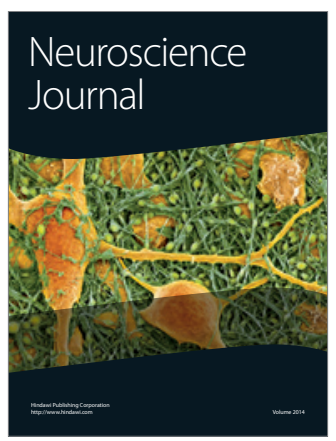

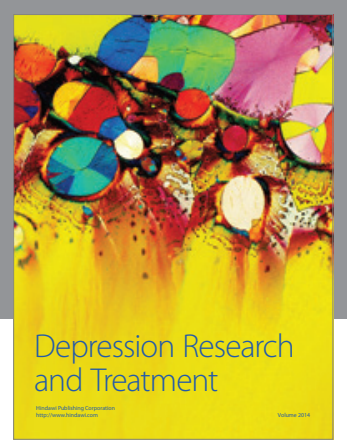
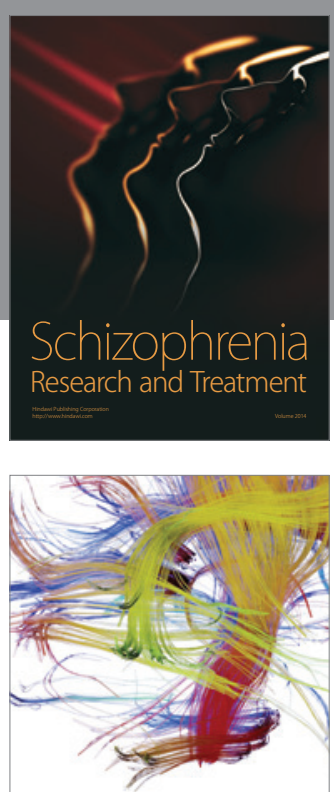

Brain Science

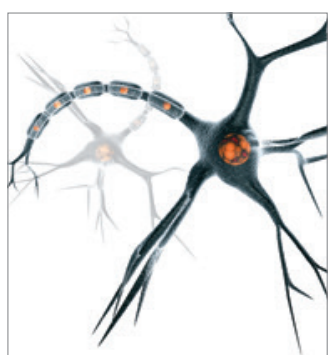

Neural Plasticity
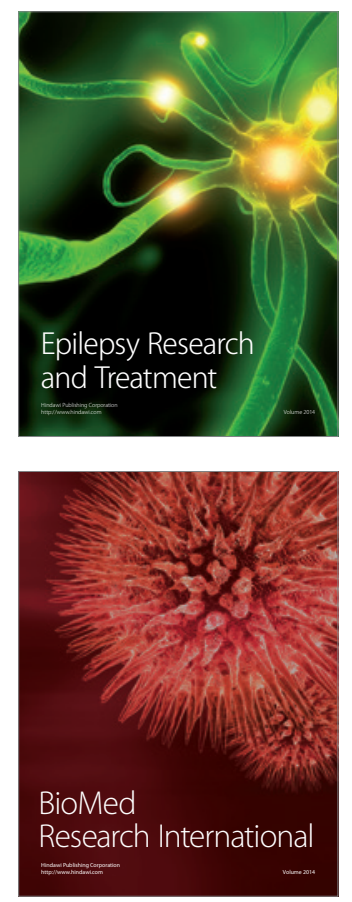

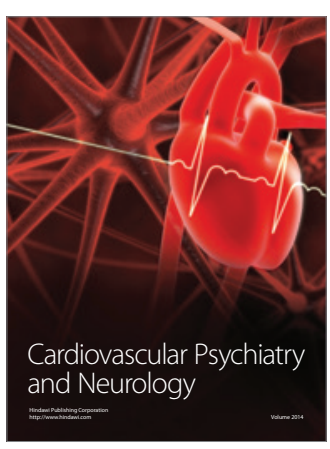

Parkinson's

Disease
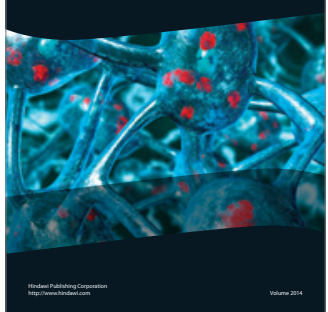